\title{
Table of constitutions, legislation, conventions and related documents
}

\section{CONSTITUTIONS AND LEGISLATION}

\author{
Angola \\ Constitution of Angola

\section{Botswana} \\ Constitution of Botswana \\ s 53 \\ s 54(1) \\ s 96(1) \\ s 96(2)
}

\section{Cameroon}

Constitution of Cameroon Article 53(3)

\section{Canada}

Charter of Rights and Freedoms

Cape Verde

Law 61/VIII/2014

Law 62/VIII/2014

\section{Eswatini}

Money Laundering and Financing of Terrorism Prevention Act 2011 s 35

\author{
Money Laundering and Financing \\ of Terrorism Prevention \\ (Amendment) Act 2016 \\ s 35 bis (1)(a)
}

104

109

\section{Ethiopia}

Proc No. 780/2013 Prevention and Suppression of Money Laundering and Financing of Terrorism

58

203, 221

205

207

207

164

Constitution of The Gambia

$$
\text { s 82(1) }
$$

s $89(2)$

203

204

111 Gambia Royal Instructions $1962 \quad 201$

\section{Ghana}

19 Anti-Money Laundering Regulations

$192011 \quad \mathbf{7 5}$

Constitution of Ghana $1957 \quad 206$

Constitution of Ghana Article 47(1) $\mathbf{2 1 6}$

Article 57(5) $\mathbf{1 6 3}$

Article $62 \quad \mathbf{2 1 6}$

Article 70(1) $\quad \mathbf{1 2 4}$

Article 72(1) 204, 206

xvii 
Article 187(2)

Article 187(3)

Article 187(5)

Article 187(6)

Article 187(7)

Article 187(12)

Article 187(13)

Article 187(14)

Article 189(2)

Economic and Organised Crime

Office Act 2010

\section{India}

Judges (Protection) Act 1985

\section{Kenya}

Anti-Corruption and Economic Crimes Act 2003

$$
\text { s } 2
$$

s 30

s 55

Companies Act 2015

s 93

Companies (Amendment) Act 2017

Constitution of Kenya

Article 10

Article 31

Article 35

Article 47(1)

Article 49(1)

Article 50

Article 79

Article 133(1)

Article 157(8)

Article 201

Article 226(3)

Article 229(2)

Article 229(4)

Article 229(5)

Article 229(6)

Article 229(8)

Article 249(2)

Article 251(1)

Article 251(2)

Article 251(5)

Power of Mercy Act 2011 s 5
119

120 s 9

129 s 13

129 s 25(1)

126, 127

128

136

126

127

75

Proceeds of Crime and Anti-Money

Laundering Act 2009

s 18

s 21

s 82

s 89

s 92(1)

Proceeds of Crime and Anti-Money Laundering (Amendment Act) 2017

166

Public Audit Act 2015

s 27

s 40

126

s 42

s 72

Witness Protection Act 2006

Witness Protection (Amendment) Act 2010

116,121

149

133

135, 136, 211

140

147, 189

189

203, 205, 207, 217

224

121

118

123

121

121

121

129

121, 127, 158

133

134

136

205

205

206

206

216

147

99

181

181

186

88

121

121

132

168

168

\section{Lesotho}

Constitution of Lesotho s 11

Money Laundering and Proceeds of Crime Act 2008

Money Laundering and Proceeds of Crime (Amendment) Act $2016 \mathbf{1 7 8}$

Mutual Legal Assistance in Criminal Matters Act 2018

\section{Liberia}

Money Laundering, Terrorist Financing, Preventive Measures and Proceeds of Crime Bill 2018

\section{Malawi}

Advisory Committee on the Granting of Pardons Act 1995

Constitution of Malawi s 42(1) s 43(1) 


s $89(2)$
s $184(1)$
s $184(3)$
Financial Crimes Act 2017
s 2
s 3
s 5
s 6
s 7
s 8
s 12
s 13
s 65
s 66
s 67
s 74
s 81
Money Laundering, Proceeds of
Serious Crime and Terrorist
Financing Act 2006
s 35
s 48
Penal Code
s 323

\section{Mauritania}

Anti-Money Laundering Law No. 2005/048

\section{Mauritius}

Banking Act 2004

Constitution of Mauritius s 10

Constitution (Amendment) Act (No. 30 of 2015)

Financial Intelligence and AntiMoney Laundering Act 2002

Financial Intelligence and AntiMoney Laundering Regulations 2018

Good Governance and Integrity Reporting Act 2015

$$
\begin{aligned}
& \text { s } 4 \\
& \text { s } 9 \\
& \text { s } 10 \\
& \text { s } 14
\end{aligned}
$$

203, 217 Mozambique

124

134 Constitution of Mozambique

109

Law No. 14/2013

185 Law No. 35/2014

78,90

78

99

142

100,101

100

91, 100

101

101

186

185

185

185

185

161

182

175

\section{Namibia}

Constitution of Namibia Article 13(1)

Article 32(3)

123,210

Article 32(9)

Article 112(3)

124

Article 127(1)

123, 127

Article 127(3)

126

Article 127(4)

133

Whistleblower Protection Act $2017 \mathbf{1 6 8}$

\section{Nigeria}

Constitution of Nigeria 1999

s $36(1)$

181

s 43

181

s $85(5)$

s $86(1)$

129

s $87(1)$

123, 125

s $87(2)$

133

128

79

$$
\begin{aligned}
& \text { s } 175(1) \\
& \text { s } 172(2) \\
& \text { s } 188
\end{aligned}
$$

204

165

s 308

165,167

Third Schedule

123,204

157 Fifth Schedule 29

Nigerian Financial Intelligence Unit Act 2018

s 2

s 5

s 7

s 11

100,101

174

s 19

102

103

Presidential Executive Order 6 of 2018

181

\section{Rwanda}

31, 191

31, 106

Presidential Order No. 27/01

\section{8}




\section{Sao Tome and Principe}

Penal Code

\section{Seychelles}

Anti-Money Laundering Act 2006

\section{South Africa}

Constitution of South Africa Chapter 9

$$
\text { s } 33
$$$$
\text { s 59(1) }
$$$$
\text { s 181(2) }
$$$$
\text { s } 182(1)
$$$$
\text { s } 188(1)
$$$$
\text { s 193(3) }
$$$$
\text { s 193(5) }
$$$$
\text { s 194(1) }
$$$$
\text { s 194(6) }
$$

Financial Intelligence Centre Act 2001

Law of Evidence Amendment Act (No. 45 of 1988)

Prevention and Combating Corrupt Activities Act (No. 12 of 2004)

$$
\text { s } 34
$$

Promotion of Access to Information Act (No. 2 of 2000)

Protected Disclosures Act (No 26 of 2000)

\section{Tanzania}

Anti-Money Laundering Act 2006

$$
\text { s } 4
$$

s 5

Prevention and Combating

Corruption Act 2007

$$
\text { s 39(1) }
$$

\section{Uganda}

Anti-Corruption Act 2009

$$
\begin{aligned}
& \text { s } 11 \\
& \text { s 44(1) }
\end{aligned}
$$

Anti-Money Laundering Act 2013

$$
\text { s } 38 \mathrm{~A}
$$

93, 96

59

$$
\text { s } 114
$$

Anti-Money Laundering (Amendment) Act 2017

Computer Misuse Act 2011

Constitution of Uganda Article 98(4) $\quad \mathbf{1 6 5}$

Article 107

163

Article 121(1)

Article 128(4)

204, 206

Article 223

Article 229

166

93

Electronic Transactions Act 2011

102

Inspectorate of Government Act 2002

$$
\text { s } 3
$$

Money Laundering (Exchange of Information) Regulations $2018 \quad \mathbf{9 6}$

Public Procurement and Disposal of Public Assets Act 2003

Trustees Incorporation Act 1939

\section{United Kingdom}

Bribery Act 2010

$$
\text { s } 7
$$

British Nationality Act 1981 sch 6

Companies Act 2006

Crime and Courts Act 2013 s $45 \quad 248$

Crime (Overseas Production Orders) Act 2019

Criminal Justice Act 1987 s 2

Proceeds of Crime Act 2002

$$
\text { s } 317
$$

Sanctions and Anti-Money Laundering Act 2018

Small Business, Enterprise and Employment Act 2015 s 81

\section{United States of America}

Foreign Corrupt Practices Act 1977 
s 76dd

s 78dd

PATRIOT Act 2010

s 311

United States Code Title

s 1956

s 1957

United States Constitution Article II

\section{Vanuatu}

Constitution of Vanuatu

Pardon (Revocation) Order No. 14 of 2015

Penal Code

214

201

\section{Zambia}

Companies Act 2017 s 3

Constitution of Zambia Article 43(3)

Article 46A

Article 96(1)

Article 97(1)

Article 121(4)

Electronic Communications and Transactions Act 2009

Financial Intelligence Centre Act 2010

Financial Intelligence Centre Act 2016

$$
\text { s } 5
$$

Prohibition and Prevention of Money Laundering Act 2001

\section{Zimbabwe}

Constitution of Zimbabwe

Preamble

Article 68

Article 112(1)

Money Laundering and Proceeds of Crime (Amendment) Act 2019
African Union Convention on
Cyber Security and Perso

Data Protection

African Union Convention on Preventing and Combating Corruption

Article 5

Article 7

Article 9

Article 11

Article 16

African Union Protocol on Amendments to the Protocol on the Statute of the African Court of Justice and Peoples' Rights

Article 28I(1)

259, 260, 261, 266,

Article 28A.2

267, 268

Article 43A(1)

Article 43A(2)

258

Article 43A(5)

Article 45(2)

Article 46A

Article 46A(4)

Article 46E

Article 46G(2)

Article 46G(3)

Article 46H(1)

Article 46H(2)

264, 265

264

265

265

266, 267

264

265,268

264

261

267

268

Article 46H(3)

268

Article 46L

263, 264

Arab Anti-Corruption Convention
Article 2

Article 3

Article 4

Article 10

Article 15

Article 20

Article 30 
European Convention on Human Rights

Article 6

Fifth EU Money Laundering

Directive

OECD Convention on Combating

Bribery of Foreign Public

Officials in International

Business Transactions

Article 4(3)

Article 5

SADC Protocol against Corruption

Article 7(1)

SADC Protocol on Mutual Legal Assistance

Article 2(1)

United Nations Convention Against Corruption

Article 8(4)

Article 8(5)

Article 10

Article 23

Article 32(2)

Article 35

Article 37

Article 46

Article 49

Article 51

Article 53

Article 54

Article 63

United Nations Convention Against

Transnational Organised Crime

Article 2

Article 6

Article 7

$14,15,35,259$

Preamble

41, 180, 217, 265

61,177

40,154

253

192

$41,183,184$

41

United Nations Convention Against

Illicit Trade in Narcotic Drugs

and Psychotropic Substances 14, 35
Vienna Convention on Diplomatic Relations 1961

\section{OTHER DOCUMENTS}

Commonwealth (Latimer House)

Principles on the Accountability

of and the Relationship between

the Three Branches of

Government

117,135

Declaration on Principles of

Freedom of Expression in Africa

151

Egmont Group of Financial Intelligence Units Charter

83,85

G20 High-Level Principles on Beneficial Ownership

Transparency

Financial Action Task Force 40

Recommendations 1990

52,53

Financial Action Task Force

Revised Recommendations 1996

53,54

Financial Action Task Force

40 Recommendations $2003 \quad 54$

Financial Action Task Force International Standards on Combating Money Laundering and the Financing of Terrorism and Proliferation 2012 (see Index)

Lima Statement on Corruption involving Vast Quantities of Assets

Mexico Declaration on Supreme Audit Authority Independence 119

United Nations Security Council Resolution 2462 (2019) 